\title{
Exfoliated syndiotactic polystyrene-graft-poly (methyl methacrylate)/montmorillonite nanocomposite prepared by solvent blending
}

\author{
Mehdi Jaymand
}

This study aims to explore an effective route for the graft copolymerization of methyl methacrylate (MMA) monomer onto syndiotactic polystyrene (sPS) using free-radical polymerization, and the effects of an organophilic montmorillonite on the final properties of graft copolymer samples. For this purpose, the chlorine groups of $\alpha$-phenyl-chloro-acetylated sPS were converted to 9-decen-1-oxy groups by a substitution nucleophilic reaction in the presence of a solvent composed of 9-decen-1-ol moiety, sodium hydride ( $\mathrm{NaH}$ ) and dry $\mathbf{N}, \mathbf{N}$-dimethylformamide. The vinyl-terminated SPS multicenter macromonomer (VsPSM) obtained was used in free-radical copolymerization with MMA monomer in a heterogeneous process to yield a graft copolymer (sPS-graftpoly(methyl methacrylate) (sPS-g-PMMA)). The structure of VsPSM and sPS-g-PMMA were determined by ${ }^{1} \mathrm{H}$ nuclear magnetic resonance and Fourier transform infrared spectroscopy. Thereafter, organophilic MMT was obtained after being treated with hexadecyl trimethyl ammonium chloride salt by an ion-exchange process. Finally, the sPS-g-PMMA/MMT nanocomposite was prepared by a solution intercalation method. X-ray diffraction and transmission electron microscopy were used to confirm nanocomposite formation. It was found that the addition of only a small amount of organoclay ( $3 \mathrm{wt} \%$ ) was enough to improve the thermal stabilities and properties of the nanocomposite.

Polymer Journal (2011) 43, 901-908; doi:10.1038/pj.2011.79; published online 7 September 2011

Keywords: free-radical polymerization; graft copolymer; macromonomer; montmorillonite; nanocomposite; poly(methyl methacrylate); syndiotactic polystyrene

\section{INTRODUCTION}

Nanocomposites are defined as materials in which the particle size of the dispersed phase is in the nanometer range in at least one dimension. Organic-inorganic hybrids based on layered inorganic compounds, such as clays and organic polymers, have been studied because of their exceptional properties, such as increased modulus, strength, reduced gas permeability and enhanced thermal stability. ${ }^{1-5}$ The dispersion of the silicate layers in the polymer matrix is improved by replacing the metal cations in the clay (such as sodium MMT; $\mathrm{Na}^{+}$-MMT) with ions bearing an aliphatic chain to compatibilize the silicate. This compatibilization enhances the silicate's interaction with the polymer by enlarging the interlayer, and the compatibilized clay is known as an organoclay. ${ }^{6-9}$ Although complete compatibility between the long aliphatic chain of the organic modifier and the polymer matrix may be desirable for better dispersion of the clay, it appears that the modification of the clay by the introduction of surfactants to obtain better compatibility is less important than the modification of the polymer matrix by the introduction of polar groups. Polymers containing polar groups capable of associative interactions, such as Lewis acid/base interactions or hydrogen bonding, lead to intercalation of polymer chains in the silicate layers. To improve the polarizability or hydrophilicity of the polymer, the functional groups in the organoclay should be short to minimize unfavorable interactions between the aliphatic chains and the polymer. ${ }^{10-12}$

Methods typically used to prepare polymer-layered inorganic nanocomposites involve the incorporation of a layered silicate into a solvent-swollen polymer (solution blending) or polymer melt (melt blending) or by addition of a modified silicate to a polymerization reaction (in situ method). ${ }^{13-18} \mathrm{~A}$ small weight percent of layered silicates that are properly dispersed throughout the polymer matrix thus create a much higher surface area for polymer/filler interactions than conventional composites can. Depending on the strength of the interfacial interactions between the polymer matrix and the layered silicate, three different types of polymer/layered silicate nanocomposites are thermodynamically achievable. If there is no affinity between the polymer and the clay, a conventional microcomposite is produced. If there is some affinity between the clay and the polymer, the polymer chains tend to penetrate between the platelets, increasing the interlayer spacing to form an intercalated microstructure. Finally, if the interaction between the clay and the polymer is very good, an exfoliated morphology can be obtained. ${ }^{19-21}$ It is generally 
accepted that exfoliation is required for enhanced permeability and mechanical properties, whereas the type of nanocomposite, either intercalated or conventional, does not seem to be important in determining the thermal properties and fire retardancy of polymer materials. ${ }^{22,23}$

Syndiotactic polystyrene (sPS) is a promising material that has been commercialized and widely studied by the academic community. sPS is regarded as a new low-cost engineering polymer with various desirable properties, such as high melting temperature $\left(270^{\circ} \mathrm{C}\right)$, fast crystallization rate, low dielectric constant, low permeability to gases and good chemical resistance. ${ }^{24-26}$ Several drawbacks can, however, restrict its practical use: its brittleness, a disadvantage in applications where mechanical properties are important; the lack of polar groups for adhesion and compatibility with other polymers; and the need to process sPS at high temperatures. Recently, several attempts have been made to improve the physical properties and processability of sPS by several procedures. One involves the syndiotactic copolymerization of styrene with a second monomer, particularly ethylene, to produce a styrene/olefin copolymer. ${ }^{27,28}$ Another modification procedure involves the preparation of functionalized sPS polymers, such as sulfonated sPS, ${ }^{29}$ acetylated $\mathrm{sPS}^{30}$ and maleic anhydride-grafted sPS. ${ }^{31}$ In addition, polymer blends also provide a way to modify sPS. ${ }^{32}$ However, because sPS usually lacks compatibility with a second polymer, blending sPS with other polymers produces weak interfacial adhesion and leads to poor mechanical properties. This problem can be solved through the use of compatibilizers, which are usually block, graft and functionalized polymers. However, it is difficult to prepare a copolymer containing a sPS block. In this respect, a graft or functionalized sPS may be the first choice to serve as compatibilizer. ${ }^{33,34}$ Graft copolymers are generally synthesized using macromonomer techniques, ${ }^{35}$ by the linkage between two different polymers through a coupling reaction ${ }^{36}$ or by the polymerization of a monomer from active sites on a polymer backbone. ${ }^{37-39}$ Macromonomers are usually referred to as reactive oligomers or polymers in which a polymerizable functional group is incorporated into the chain end(s). Macromonomers can be synthesized using various methods, including anionic, cationic and radical polymerization, as well as the chemical modification of polymer ends. The use of macromonomers provides a facile route to control the molecular structure of polymers, including crosslinked and branched copolymers, such as comb, star, brush and graft types. ${ }^{40,41}$

In our previous studies, we investigated the synthesis and characterization of a syndiotactic polystyrene-grafte-[polystyrene-block-poly ( $p$-methylstyrene)]/MgAl-layered double-hydroxide nanocomposite using polymer intercalation from the solution into the MgAl-layered double hydroxide. ${ }^{13}$ At least two important factors need to be considered to achieve the homogeneous dispersion of the clay or layered double hydroxide layers in the sPS or its graft copolymer hybrids. First, the surfactant should be intercalated between the silicate layers of the clay by ionic bonding. Second, the hydrophobic tail of the surfactant molecule should interact or be partially compatible with sPS or its graft copolymer molecules. We report here our preliminary investigations on the solvent-blending preparation of sPS-graft-poly (methyl methacrylate) (sPS-g-PMMA) nanocomposite with alkylammonium-modified MMT. For this purpose, a vinyl-terminated sPS multicenter macromonomer (VsPSM) was synthesized by reacting $\alpha$-phenyl-chloro-acetylated sPS ( $\alpha$-ph-ch-sPS) with 9-decen-1-oxy derived from a 9-decen-1-ol moiety. The resulting multicenter macromonomer was subsequently copolymerized by free-radical polymerization to yield the graft copolymer (sPS-g-PMMA) in the presence of methyl methacrylate (MMA) monomer. Thereafter, MMT was organi- cally modified using an ammonium salt. This clay was used to prepare an sPS-g-PMMA/MMT nanocomposite by a solvent-blending method.

\section{EXPERIMENTAL PROCEDURE}

\section{Materials}

$\mathrm{Na}^{+}$-MMT was obtained from Southern Clay Products (Gonzales, TX, USA) under the trade name Cloisite $\mathrm{NaC}$, which possesses the idealized chemical formula $\mathrm{Na}_{0.33}\left[\mathrm{Mg}_{0.33} \mathrm{Al}_{11.67} \mathrm{Si}_{4} \mathrm{O}_{10}\right](\mathrm{OH})_{2}$ and a cation exchange capacity of $95 \mathrm{meq} 100 \mathrm{~g}^{-1}$. The polymer $\alpha$-ph-ch-sPS was prepared in our laboratory. MMA monomer was supplied by Merck (Darmstadt, Germany) and was distilled under vacuum before use. Toluene (Merck) was dried by refluxing over sodium and distilled under argon atmosphere before use. 2,2'-Azobis (isobutyronitrile) from Merck was recrystallized in ethanol at $50{ }^{\circ} \mathrm{C}$ before use. Sodium hydride $(\mathrm{NaH})$, 9-dcen-1-ol and hexadecyl trimethyl ammonium chloride were supplied by Merck and used without further purification. All other reagents were supplied by Merck and purified according to the literature.

\section{Introduction of vinyl terminal group to the $\alpha$-ph-ch-sPS}

In a three-neck round-bottom flask equipped with a condenser, dropping funnel, gas inlet/outlet and a magnetic stirrer, $100 \mathrm{mg}(0.64 \mathrm{mmol})$ of 9-decen1 -ol was dissolved in anhydrous $\mathrm{N}, \mathrm{N}$-dimethylformamide $(5 \mathrm{ml})$ and added under argon atmosphere to $23 \mathrm{mg}(0.90 \mathrm{mmol})$ of hexane-washed $\mathrm{NaH}$ (from $60 \%$ suspension in oil). The mixture was stirred for $30 \mathrm{~min}$, and $1 \mathrm{~g}$ of $\alpha$ phenyl-chloro-acetylated sPS was then added under an argon atmosphere and refluxed for $24 \mathrm{~h}$ at room temperature. The reaction was terminated by pouring the contents of the flask into a large amount of acidic methanol. The obtained white solid was filtered and dried under vacuum.

\section{Synthesis of sPS-g-PMMA via free-radical polymerization}

sPS containing undecen-10-enyl-2-phenyl acetate, multicenter macromonomer $(0.50 \mathrm{~g})$, MMA monomer $(3 \mathrm{~g}, 30 \mathrm{mmol})$ and toluene $(10 \mathrm{ml})$ were placed in a typical experiment flask. An initiator (2,2'-azobisisobutyronitrile) was added to the mixture, and the reaction mixture was thoroughly flushed with argon for $20 \mathrm{~min}$. Thereafter, the mixture was heated to $70{ }^{\circ} \mathrm{C}$ in an oil bath and stirred under flowing argon to complete the polymerization. During the reaction, the viscosity of the mixture was observed to gradually increase. After $12 \mathrm{~h}$, the mixture was dissolved in $10 \mathrm{ml}$ of $\mathrm{CH}_{2} \mathrm{Cl}_{2}$ and precipitated in $50 \mathrm{ml}$ of hexane for $24 \mathrm{~h}$ and then reprecipitated from $\mathrm{CH}_{2} \mathrm{Cl}_{2}$ into $50 \mathrm{ml}$ of methanol. The crude product was then dried under vacuum. The obtained powder was extracted with acetone in a Soxhlet apparatus (Tabriz, Iran) for $24 \mathrm{~h}$ to remove the PMMA homopolymer.

\section{Organic modification of the MMT}

Organophilic MMT (O-MMT) was prepared by cation exchange between $\mathrm{Na}^{+}$ in clay galleries and hexadecyl trimethyl ammonium cations in aqueous solution. MMT was first dispersed in deionized water under ultrasound for $20 \mathrm{~min}$. Aqueous solution (3 wt\%) of modifier (hexadecyl trimethyl ammonium chloride salt) was prepared in deionized water separately, and was added to the clay dispersion in an amount slightly larger higher than the cation exchange capacity of MMT. The resulting suspension was intensively stirred for $10 \mathrm{~h}$ at $60^{\circ} \mathrm{C}$, and was then filtered and washed with deionized water until it was free of chloride ions, according to a test with an aqueous $\mathrm{AgNO}_{3}$ solution. The final product was dried in a vacuum oven at room temperature for $48 \mathrm{~h}$ and ground into a powder.

\section{Preparation of sPS-g-PMMA/MMT nanocomposite by solvent blending}

The sPS-g-PMMA/MMT nanocomposite was prepared by solution intercalation. For this purpose, a certain amount $(0.5 \mathrm{~g})$ of sPS-g-PMMA was dissolved in $30 \mathrm{ml} \mathrm{CHCl}$. Thereafter, a certain amount of O-MMT (3 wt\%) was first dispersed in $30 \mathrm{ml} \mathrm{CHCl}_{3}$ under ultrasonic treatment for $20 \mathrm{~min}$. The clay suspension was then slowly added to the polymeric solution under vigorous stirring. The resulting suspension was intensively stirred for $5 \mathrm{~h}$ at $80^{\circ} \mathrm{C}$. Thereafter, the mixture was poured into $200 \mathrm{ml}$ methanol for rapid precipitation. The precipitate was filtered and dried at $50{ }^{\circ} \mathrm{C}$ under vacuum for 2 days. 


\section{Characterization}

Fourier transform infrared (FTIR) spectra of the samples were obtained on a Shimadzu 8101M FTIR (Shimadzu, Kyoto, Japan). The samples were prepared by grinding the dry powders with $\mathrm{KBr}$ and compressing the mixture into disks. The disks were stored in a desiccator to avoid moisture absorption. The spectra were recorded at room temperature. ${ }^{1} \mathrm{H}$ and ${ }^{13} \mathrm{C}$ nuclear magnetic resonance (NMR) spectra were obtained at $25^{\circ} \mathrm{C}$ using an FT-NMR Bruker spectrometer (Bruker, Ettlingen, Germany). The sample for NMR spectroscopy was prepared by dissolving about $10 \mathrm{mg}$ of products in $5 \mathrm{ml}$ of deuterated chloroform. ${ }^{1} \mathrm{H}$ NMR and ${ }^{13} \mathrm{C}$-NMR measurements were taken at frequencies of 400 and $100 \mathrm{MHz}$, respectively. Transmission electron microscopy (TEM) was performed using a Philips EM208 microscope (Phillips, Eindhoven, The Netherlands) with a $100-\mathrm{kV}$ accelerating voltage. X-ray diffraction (XRD) spectra were obtained with a Siemens D 5000 (Aubrey, TX, USA) X-ray generator (CuK $\alpha$ radiation with $\lambda=1.5406 \AA$ ) with a $2 \theta$ scan range of $2-25^{\circ} \mathrm{C}$ at room temperature. The thermal properties of the copolymer and the nanocomposite were obtained with a TGA-PL STA 1640 (Polymer Laboratories, Shropshire, UK). About $10 \mathrm{mg}$ of the sample was heated between 25 and $600{ }^{\circ} \mathrm{C}$ at a rate of $10^{\circ} \mathrm{C} \mathrm{min}^{-1}$ under flowing nitrogen. Differential scanning calorimetry (DSC) analyses were performed with a NETZSCH-DSC 200 F3 Maia (Selb, Germany). The sample was first heated to $200^{\circ} \mathrm{C}$ and then allowed to cool for $5 \mathrm{~min}$ to eliminate the thermal history. Thereafter, the sample was reheated to $200{ }^{\circ} \mathrm{C}$ at a rate of $10^{\circ} \mathrm{C} \mathrm{min}^{-1}$. The entire test was performed under nitrogen purging at a flow rate of $50 \mathrm{ml} \mathrm{min}^{-1}$.

\section{RESULTS AND DISCUSSION}

As illustrated in Scheme 1, at first, to produce active sites on sPS, 9-decen-1-ol was added to sPS. Then, to generate active sites on sPS and free-radical copolymer after the $\alpha$-phenyl-chloro-acetylation of sPS, 9-decen-1-oxy was reacted with $\alpha$-ph-ch-sPS to produce the VsPSM. In this study, the vinyl terminal group of undecen-10-enyl-2phenyl acetate formed the active sites for the MMA monomer grafts via free-radical polymerization. To prepare a polymer clay nanocomposite, the gallery space must be large and sufficiently organophilic to permit the entry of the organic polymer. The usual treatment is to replace the inorganic ions of clay by an ammonium ion that contains at least one long alkyl chain. The identity of the cation can be critical to the formation of the nanocomposite. The method of preparation to be used also affects the choice of the organically modified clay.

\section{Synthesis of macromonomer (VsPSM)}

Figure 1 shows the FTIR spectra of the $\alpha$-phenyl-chloro-acetylated sPS (a) and the VsPSM (b). The FTIR spectra of the $\alpha$-ph-ch-sPS sample show an absorption band at $1736 \mathrm{~cm}^{-1}$ attributed to the carbonyl stretching bond. The characteristic absorption bands due to the stretching vibrations of $\mathrm{C}-\mathrm{H}$ (aliphatic and aromatic) appear in the $3100-2850 \mathrm{~cm}^{-1}$ region, $\mathrm{C}=\mathrm{C}$ stretching vibrations appears at 1584 and $1507 \mathrm{~cm}^{-1}$, and $\gamma(\mathrm{C}-\mathrm{H})$ in the aromatic ring appears at 757 and $694 \mathrm{~cm}^{-1}$.

The FTIR spectra of the VsPSM show similar absorption bands with minor differences. The characteristic absorption band due to the stretching vibrations of carbonyl group is shifted to $1607 \mathrm{~cm}^{-1}$ from $1736 \mathrm{~cm}^{-1}$. This is because of the fact that the chlorine group in the $\alpha$ ph-ch-sPS sample is an electron withdrawer compared with that of the $-\mathrm{CH}_{2} \mathrm{O}$ group in the VsPSM. In addition, after introducing 9-decen1 -oxy to the $\alpha$-ph-ch-sPS sample, an increase is observed in the intensity of the aliphatic $\mathrm{C}-\mathrm{H}$ stretching vibrations compared with that of the aromatic $\mathrm{C}-\mathrm{H}$ stretching vibrations. These FTIR spectra assignments verify that the 9-decen-1-oxy was introduced into the $\alpha$ ph-ch-sPS, and thus the VsPSM was successfully synthesized.

Additional evidence for the synthesis of the VsPSM was also obtained from ${ }^{1} \mathrm{H}-\mathrm{NMR}$ data. A comparison of the ${ }^{1} \mathrm{H}-\mathrm{NMR}$ spectrum of $\alpha$-ph-ch-sPS with the ${ }^{1} \mathrm{H}$-NMR spectrum of VsPSM indicates that during the reaction the chemical shifts of vinyl methylene $(\mathrm{g})$ and methine (h) protons appear at 5.07 and 5.78 p.p.m., respectively, as well as the chemical shifts of $-\mathrm{CH}_{2} \mathrm{O}$ protons (e) and phenyl ring<smiles>CCC(CC(CC(CC(=O)c1ccccc1)c1ccccc1)c1ccccc1)c1ccc(C(=O)C(Cl)c2ccccc2)cc1</smiles>

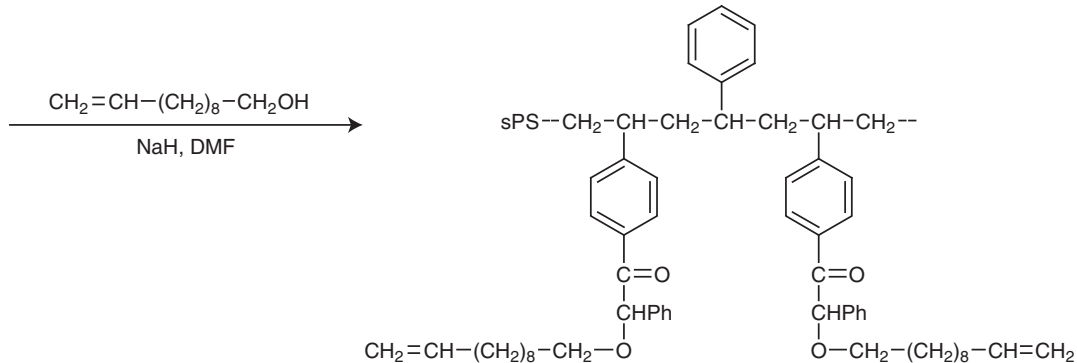

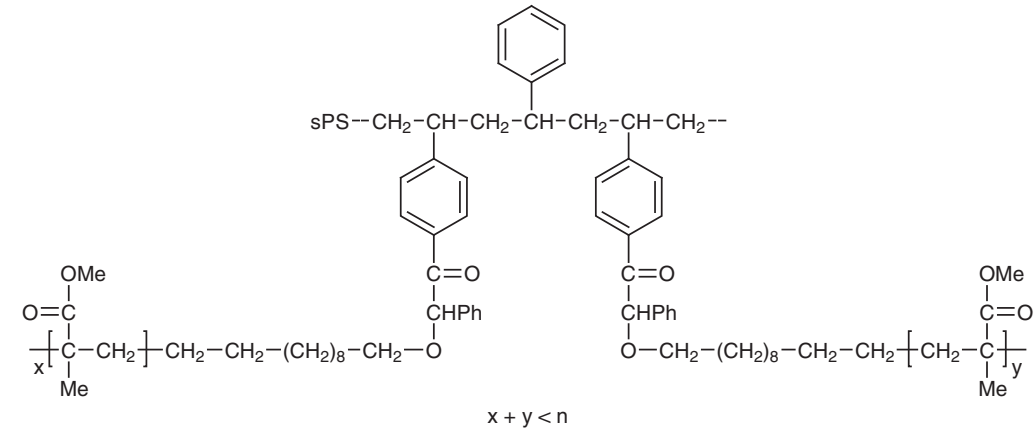

Scheme 1 Synthetic route of the vinyl-terminated syndiotactic polystyrene multicentre macromonomer (VsPSM) and graft copolymerization of methyl methacrylate onto syndiotactic polystyrene. 


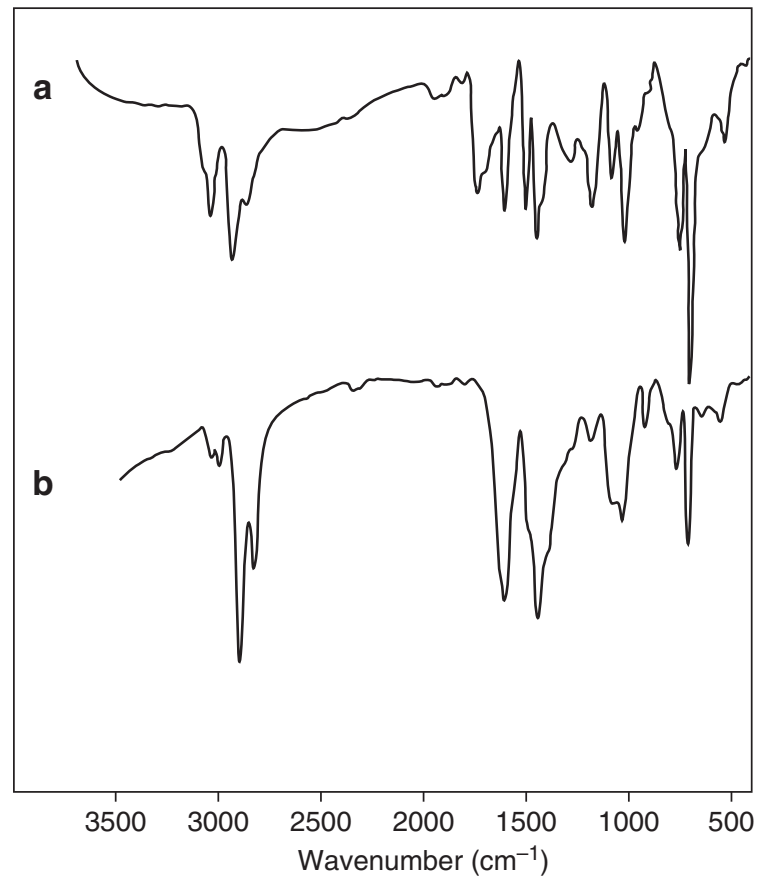

Figure 1 Fourier transform infrared spectra of $\alpha$-phenyl-chloro-acetylated syndiotactic polystyrene (a) and vinyl-terminated syndiotactic polystyrene multicenter macromonomer (b).

protons (i, $j$ and $k$ ) at 3.46 and 6.62-7.34 p.p.m., respectively, are clearly observed. Furthermore, before introducing undecen-10-enyl-2phenyl acetate to the sPS, the ratio of aliphatic hydrogens to aromatic hydrogens is 3:5; after introducing undecen-10-enyl-2-phenyl acetate to the sPS, an increase $(0.2107: 0.2084 \sim 1.01: 1)$ in the ratio of the integration intensity of aliphatic hydrogens ( $a, b$ and $c$ ) of the macromonomer to that of aromatic hydrogens ( $i, j$ and $k$ ) of the macromonomer can be attributed to the fact that the undecen10-enyl-2-phenyl acetate was introduced into the sPS, and that the VsPSM was successfully synthesized. The ${ }^{1} \mathrm{H}-\mathrm{NMR}$ spectrum of VsPSM indicates that all of the chlorine groups in the $\alpha$-ph-ch-sPS were substituted with 9-decen-1-oxy because the chemical shift of $-\mathrm{CHPhCl}$ at 3.93 p.p.m. completely disappears, and the new peak for -OCHPh (f) group is observed at 3.86 p.p.m. The characteristic signal at 7.26 p.p.m. is related to the $\mathrm{CDCl}_{3}$.

To calculate the extent of the presence of the vinyl terminal group for sPS, the following method was adopted. Before the introduction of undecen-10-enyl-2-phenyl acetate into the sPS, the ratio of aliphatic hydrogens to aromatic hydrogens was 3:5; after the introduction of undecen-10-enyl-2-phenyl acetate, this ratio changed to 0.2107:0.2084 1.01:1. Thus, $((17 \times X)+((1-X) \times 3)):((9 \times X)+(1-X) \times 5)$ $\sim 1.01: 1$, where $X$ is the substitution percent of undecen-10-enyl2-phenyl acetate, 17 is the number of aliphatic hydrogens in the substituted part (1.23-1.78 p.p.m.), 3 is the number of aliphatic hydrogens in the unsubstituted part, 9 is the number of aromatic hydrogens in the substituted part (6.62-7.34 p.p.m.) and 5 is the number of aromatic hydrogens in the unsubstituted part. In Figure $2 b$, by solving the equation, it can be seen that $X=20.58 \%$.

\section{Characterization of graft copolymer}

The FTIR spectrum of the sPS-g-PMMA (Figure 3) shows the characteristics of the functional groups present in the synthesized a
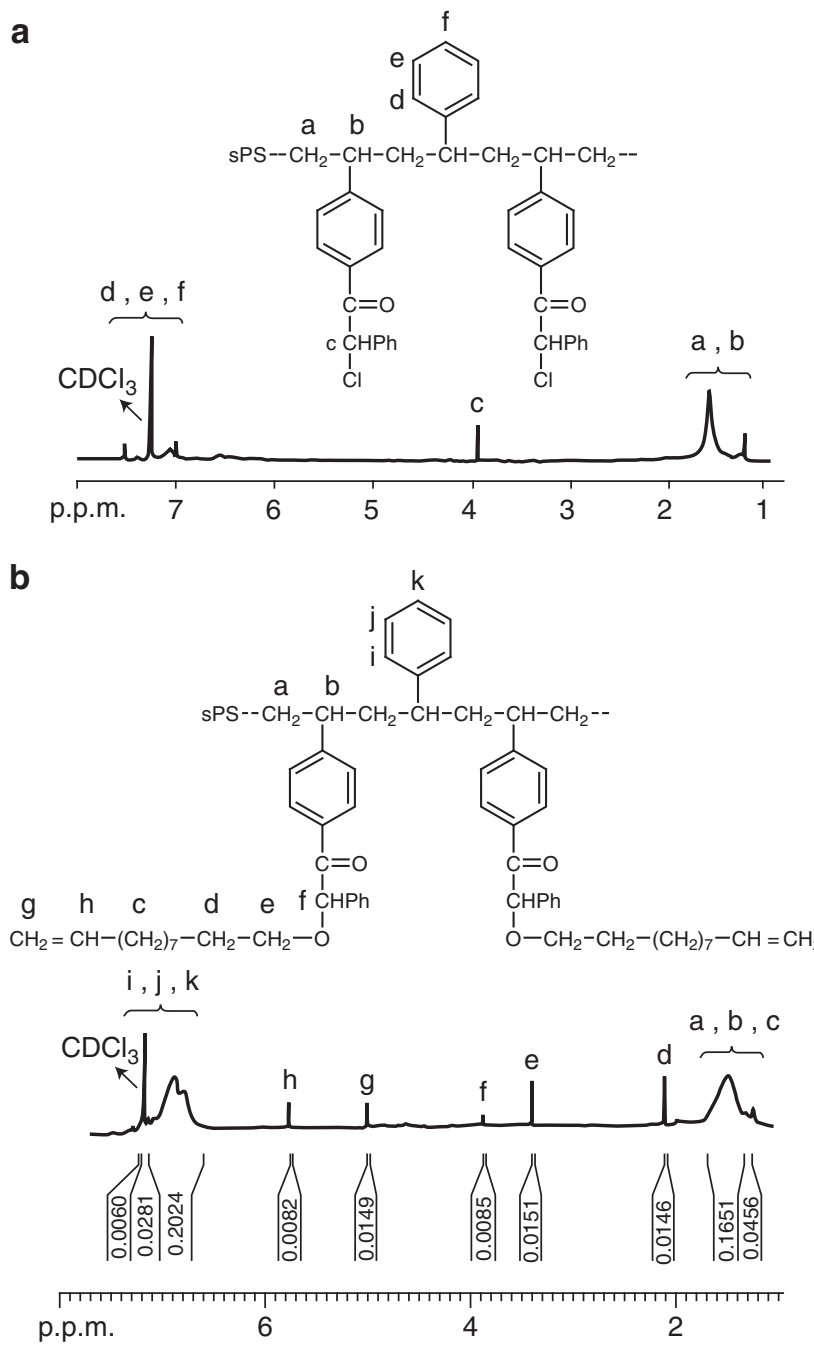

Figure $2{ }^{1} \mathrm{H}$ nuclear magnetic resonance spectra of $\alpha$-phenyl-chloroacetylated syndiotactic polystyrene (a) and vinyl-terminated syndiotactic polystyrene multicenter macromonomer (b).

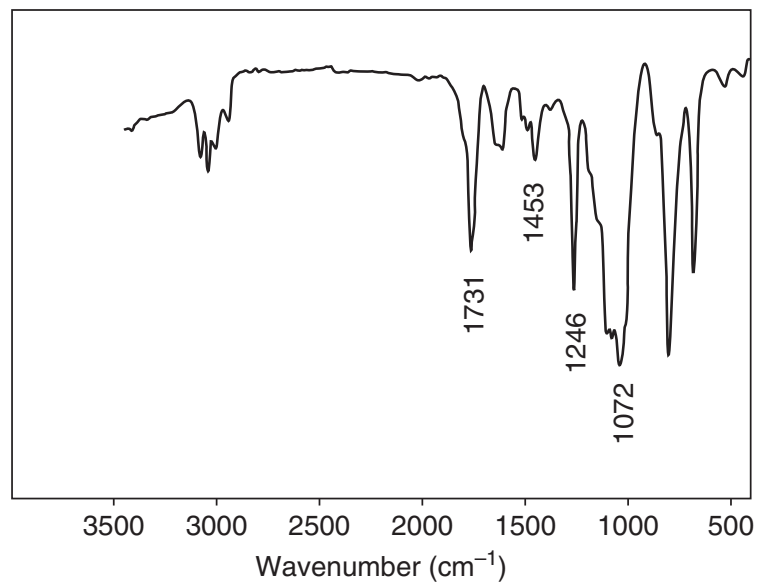

Figure 3 Fourier transform infrared spectra of syndiotactic polystyrene-graftpoly(methyl methacrylate).

graft copolymer. The fingerprint characteristic vibrational bands of PMMA appear at $1731 \mathrm{~cm}^{-1} v(\mathrm{C}=\mathrm{O})$ and $1453 \mathrm{~cm}^{-1} v(\mathrm{C}-\mathrm{O})$. The band at $1246 \mathrm{~cm}^{-1}$ is assigned to the torsion of the methylene group 
$\left(-\mathrm{CH}_{2}\right)$ of sPS-g-PMMA, the band at $1072 \mathrm{~cm}^{-1}$ corresponds to the vibration of the ester group $(\mathrm{C}-\mathrm{O})$ of PMMA segments and the characteristic absorption bands in the $3100-2900 \mathrm{~cm}^{-1}$ region are due to the $\mathrm{C}-\mathrm{H}$ stretching vibrations of the sPS-g-PMMA copolymer.

The ${ }^{1} \mathrm{H}-\mathrm{NMR}$ and ${ }^{13} \mathrm{C}-\mathrm{NMR}$ spectra of the sPS-g-PMMA are shown in Figures $4 \mathrm{a}$ and $\mathrm{b}$. The ${ }^{1} \mathrm{H}-\mathrm{NMR}$ spectra of the sPS-gPMMA show the major characteristic resonance of $-\mathrm{OOCH}_{3}$ at $\delta=3.58$ p.p.m., which is related to the $-\mathrm{COOCH}_{3}$ groups of the PMMA segments; the peaks at $\delta=0.75-1.15$ p.p.m., which are related to the methyl carbon $\left(-\mathrm{CH}_{3}\right)$ groups of the PMMA segments, methylene $\left(-\mathrm{CH}_{2}\right)$ and methine $(-\mathrm{CH})$ protons of sPS-g-PMMA, show peaks in the range 1.35-2.15 p.p.m., and the peaks at $\delta=4.75-$ 5.25 p.p.m. are related to the vinyl protons that formed on the ends of PMMA chains.

The chemical structure of the graft copolymer was further examined by ${ }^{13} \mathrm{C}-\mathrm{NMR}$. The ${ }^{13} \mathrm{C}-\mathrm{NMR}$ spectrum of the sPS-g-PMMA (Figure $4 \mathrm{~b}$ ) shows all of the peaks corresponding to carbon atoms in different environments in the copolymer structure. The quaternary carbon $(-\mathrm{C}-)$, methoxy carbon $\left(-\mathrm{OCH}_{3}-\right)$ and methylene carbon $\left(-\mathrm{CH}_{2}-\right)$ gave characteristic peaks around $43.8,51.2$ and 52.9 p.p.m., respectively. The chemical shifts at 16.6 and 18.7 p.p.m. are assigned to methyl carbon $\left(-\mathrm{CH}_{3}\right)$, and the carbonyl carbon group $(-\mathrm{C}=\mathrm{O})$ produced characteristic peaks around 181.8 and 185.3 p.p.m. The
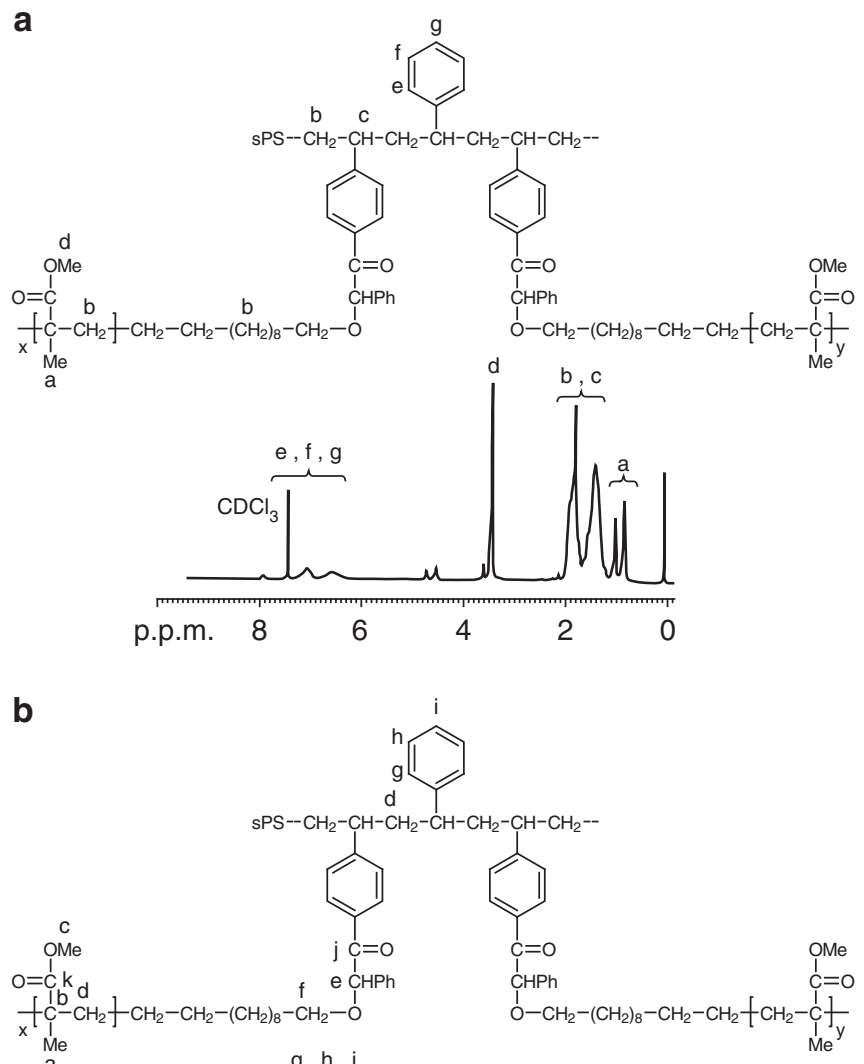

Me

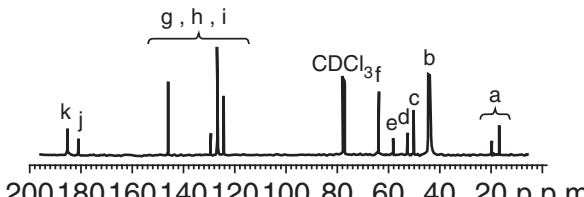

20018016014012010080604020 p.p.m.

Figure $4{ }^{1} \mathrm{H}$ nuclear magnetic resonance spectra (a) and ${ }^{13} \mathrm{C}$ nuclear magnetic resonance spectra (b) of syndiotactic polystyrene-graft-poly(methyl methacrylate). chemical shifts at $124-144$ p.p.m. are assigned to $-\mathrm{C}=\mathrm{C}-$ (aliphatic or aromatic), and the characteristic peaks around 74-78 p.p.m. are assigned to the $\mathrm{CDCl}_{3}$ solvent. Moreover, the chemical shifts at 57.8 and 63.4 p.p.m. are attributed to $-\mathrm{OCHPh}$ and $-\mathrm{CH}_{2} \mathrm{O}$ groups, respectively.

\section{Preparation of organophilic MMT}

$\mathrm{Na}^{+}$-MMT is the most commonly used layered silicate because of its natural occurrence and beneficial properties (high cation exchange capacity, high surface area and large aspect ratio). ${ }^{42}$ The structure of $\mathrm{Na}^{+}$-MMT is comprised of layers made up of one octahedral alumina sheet sandwiched between two tetrahedral silica sheets. About one in six of the aluminum ions in the octahedral layers of $\mathrm{Na}^{+}-\mathrm{MMT}$ is isomorphously substituted in the sheet structure by magnesium or other divalent ions; this results in negative charges that are in turn counterbalanced by $\mathrm{Na}^{+}$cations residing in the interlayer space. ${ }^{23}$ The $\mathrm{Na}^{+}$ions in the gallery space can be ion exchanged by an organic cation (surfactant) to yield a surfactant-modified clay.

Figure 5 shows the FTIR spectra of unmodified MMT (a) and modified MMT (b). In the spectra of both the modified and unmodified clays, the intense peak at $1045 \mathrm{~cm}^{-1}$ and the two bands at 467 and $525 \mathrm{~cm}^{-1}$ are assigned to $\mathrm{Si}-\mathrm{O}$ stretching bonds and $\mathrm{Si}-\mathrm{O}$ bending bonds, respectively. The bands around 1637 and $3443 \mathrm{~cm}^{-1}$ are due to the hydroxyl groups in the clay. The other additional bands observed in the spectra of the modified clays arise from surfactant stretching or bending bonds. The FTIR spectra of the modified clay show an absorption band at $1467 \mathrm{~cm}^{-1}$ attributed to $\mathrm{C}-\mathrm{H}$ bending bonds. In addition, the intercalation of modifier instead of sodium ions can be confirmed by the $\mathrm{C}-\mathrm{H}$ stretching vibrations at 2855 and $2925 \mathrm{~cm}^{-1}$.

Figure 6 shows the XRD patterns of the parent MMT (a) and the organo-modified MMT (b). The $d_{001}$-spacing was calculated from the peak positions using Bragg's law: $d=\lambda /(2 \sin \theta)$. An increase in the basal spacing $\left(d_{001}\right)$ of the modified clay is observed after the insertion of the surfactant. More specifically, the pristine MMT shows a $d_{001^{-}}$ spacing of $12.6 \AA$, which corresponds to an interlayer spacing $D=12.6-$ $9.6=3 \AA$, where $9.6 \AA$ is the thickness of an individual clay sheet. In the

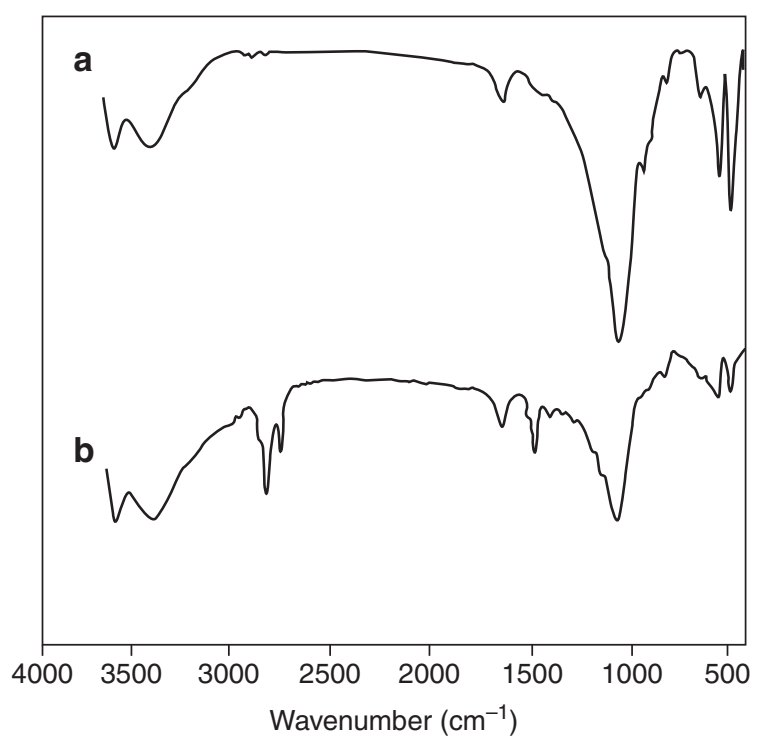

Figure 5 Fourier transform infrared spectra of unmodified montmorillonite (MMT) (a) and modified MMT (b). 


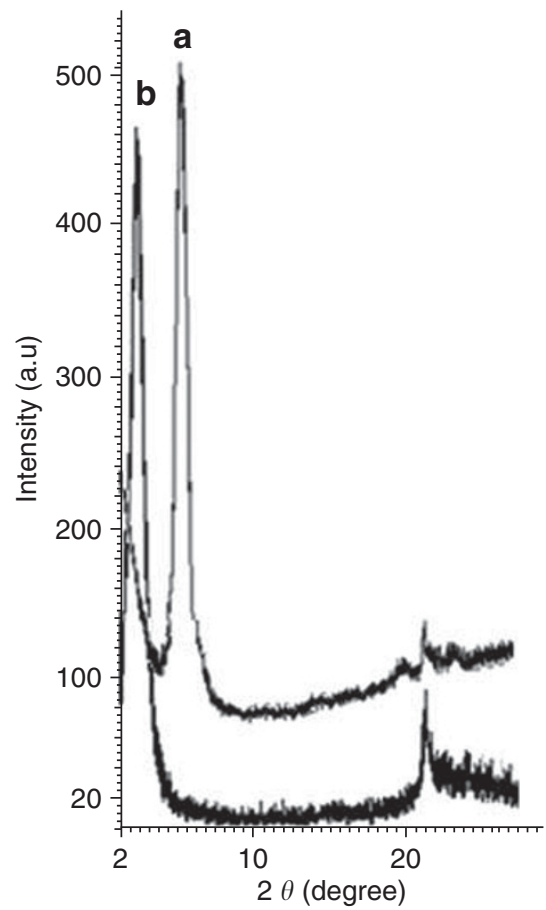

Figure 6 X-ray diffraction patterns of the parent montmorillonite (MMT) (a) and the organo-modified MMT (b).

case of the organoclay, the basal spacing becomes $18.38 \AA$, with a corresponding interlayer space $D=8.66 \AA$.

\section{Structural characterization of the sPS-g-PMMA/MMT nanocomposite}

The synthesis of the sPS-g-PMMA/MMT nanocomposite was achieved by the intercalation of sPS-g-PMMA onto modified MMT clay through a solvent-blending technique. XRD is often used to characterize the structure types of polymer/layered inorganic filler nanocomposites, that is, intercalated and/or exfoliated structures, because the peaks change with the gallery height of the inorganic fillers. The formation of an intercalated structure should result in a decrease in $2 \theta$, which would indicate an increase in the $d$-spacing. The formation of an exfoliated structure usually results in the complete loss of registry between the clay layers, and thus no peak can be seen in the XRD trace. In some cases, a disordered immiscible system is obtained, which also shows no peaks; hence, the absence of an XRD peak cannot be taken as definitive evidence of the formation of an exfoliated nanocomposite, and additional evidence, usually TEM images, is required. ${ }^{13,43}$ The XRD patterns of the sPS-g-PMMA/ MMT nanocomposite in the range of $2 \theta=2-25^{\circ}$ is shown in Figure 7. For the nanocomposite, no peaks are observed, which could mean that either an exfoliated or an immiscible nanocomposite has been formed.

$\mathrm{XRD}$ is a powerful technique used to observe the extent of ordered or disordered silicate dispersion structures in the sPS-g-PMMA nanocomposite. However, XRD does not provide information concerning the spatial distribution of silicate layers in the polymer matrix or the shape of the hybrid because all of its data are 'averaged' over the whole of the sample. TEM is required to complement this information to enable the evaluation of the dispersion of clay in the polymer matrix. Usually, both low-magnification images, which show whether the silicate layers are well dispersed, and high-magnification images, which allow for the identification of morphology, are necessary. The

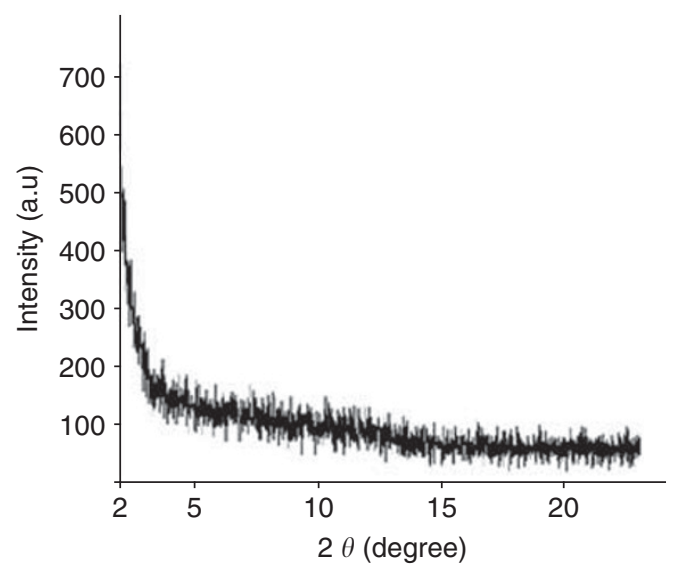

Figure 7 X-ray diffraction patterns of the syndiotactic polystyrene-graftpoly(methyl methacrylate)/montmorillonite nanocomposite.

morphologies of the sPS-g-PMMA/MMT nanocomposite with $3 \mathrm{wt} \%$ O-MMT loading are shown in Figure 8. Light regions represent the polymer matrix, and dark lines indicate silicate layers. It is obvious from the low-magnification image (Figure 8a) that the clay was well dispersed throughout the polymer and that a nanocomposite was indeed formed. At higher magnification (Figure 8b), the delamination of O-MMT into individual layers can be clearly seen in the filler particle region. TEM images provided evidence for the formation of an exfoliated structure for sPS-g-PMMA/MMT nanocomposites, in accordance with the results obtained by XRD studies.

\section{Glass transition of the sPS-g-PMMA and sPS-g-PMMA/MMT nanocomposite}

To obtain additional evidence that PMMA was chemically grafted onto the sPS backbone, DSC was used. As expected, both the melting point $\left(T_{\mathrm{m}}\right)$ and glass transition temperature $\left(T_{\mathrm{g}}\right)$ of the graft copolymer are affected by the side chains. The random incorporation of non-crystallizable monomer units into the backbone of a semicrystalline polymer has a marked effect on the thermodynamics and kinetics of crystallization. Relative to the behavior observed with the homopolymer, the crystallizable copolymer usually exhibits a low melting temperature, low degree of crystallinity and a significant decrease in the overall rate of crystallization. ${ }^{13,34}$ The $T_{\mathrm{g}}$ data also help in understanding the effects of constituent groups on the movement of polymer chains. It is clear from Figure 9 that the $T_{\mathrm{m}}$ and $T_{\mathrm{g}}$ values of the modified polymers depend on the graft copolymerization. This is easy to understand because the substituent groups reduce the mobility of the polymer chain and thereby increase the $T_{\mathrm{g}}$ values.

The DSC curves for sPS, sPS-g-PMMA and the sPS-g-PMMA/ MMT nanocomposites are shown in Figure 9. For pure sPS, $T_{\mathrm{g}}$ and $T_{\mathrm{m}}$ were observed around 98.4 and $270{ }^{\circ} \mathrm{C}$, respectively. The $T_{\mathrm{g}}$ and $T_{\mathrm{m}}$ values of sPS-g-PMMA are 102.6 and $246^{\circ} \mathrm{C}$, respectively. The $T_{\mathrm{g}}$ values of sPS-g-PMMA are slightly higher than those of pure sPS. In addition, the $T_{\mathrm{g}}$ slightly increased in the presence of PMMA segments by impeding chain flexibility. The $T_{\mathrm{g}}$ and $T_{\mathrm{m}}$ values of the sPS-g-PMMA/MMT nanocomposite (3 wt \% MMT) are 104.8 and $248^{\circ} \mathrm{C}$, respectively, which are slightly higher than those of pure sPSg-PMMA. This is tentatively ascribed to the confinement of the polymer chains adjacent to the MMT layers, which prevents the segmental motion of the polymer chains. Because O-MMT layers are dispersed in the polymer matrix at the nanoscale, strong interfacial forces are created between the silicate layers and the polymer chains, 

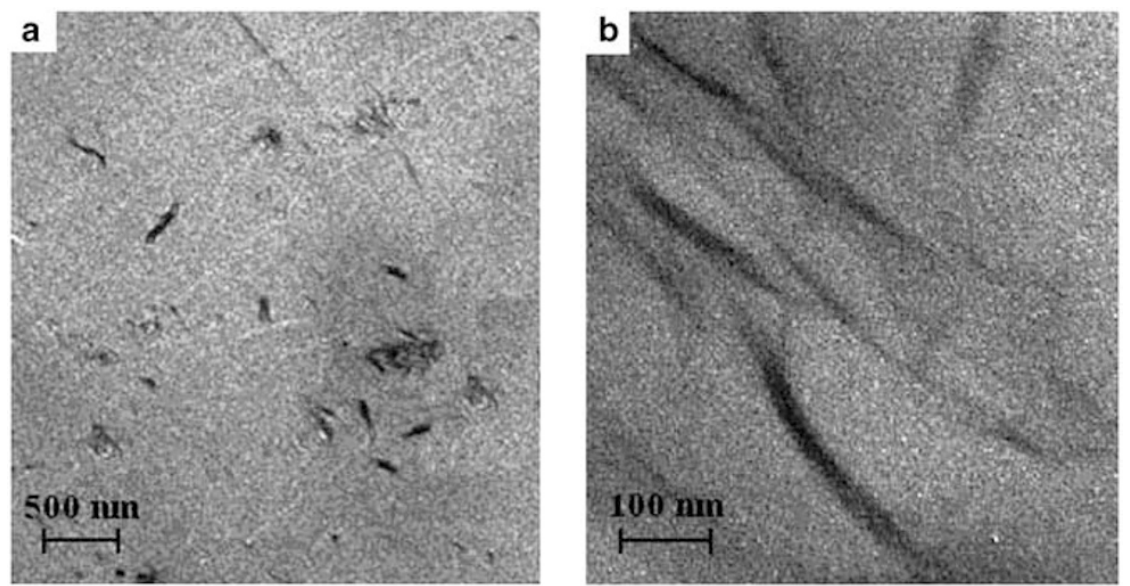

Figure 8 Transmission electron microscope images of the syndiotactic polystyrene-graft-poly(methyl methacrylate)/montmorillonite nanocomposite at low magnification (a) and at high magnification (b).

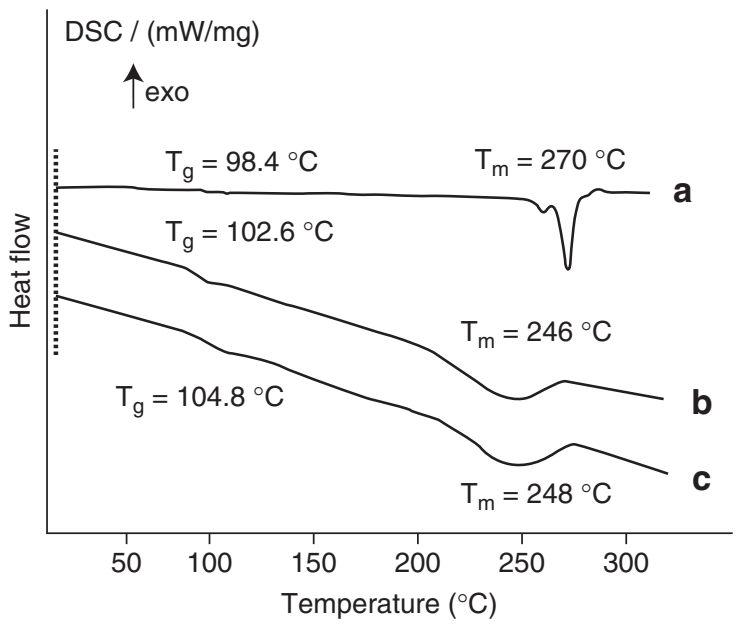

Figure 9 Differential scanning calorimeter traces of the neat syndiotactic polystyrene (sPS) (a), sPS-graft-poly(methyl methacrylate) (sPS-g-PMMA; b) and the SPS-g-PMMA/montmorillonite nanocomposite (c).

which may confine the movement of chain segments, resulting in an increase of $T_{\mathrm{g}}$ and $T_{\mathrm{m}}$. As a kind of inorganic material, the excellent thermal stability of MMT can be fully displayed; this leads to an improvement in the heat resistance of the nanocomposites, which is obvious upon the addition of O-MMT.

\section{Thermal stability of the sPS-g-PMMA/MMT nanocomposite}

The thermal properties of the neat copolymer and the nanocomposite were studied by thermogravimetric analysis. Figure 10 shows the thermogravimetric analysis traces of the pure sPS-g-PMMA copolymer and the sPS-g-PMMA/MMT nanocomposite. It is evident that the neat sPS-g-PMMA undergoes a two-step decomposition; the first step corresponds to the decomposition of the PMMA chain (275$380^{\circ} \mathrm{C}$ ), whereas the second step is associated with sPS chain scission $\left(380-480^{\circ} \mathrm{C}\right)$. The sPS-g-PMMA/MMT nanocomposite also undergoes such degradation steps with an initial additional step because of the previous decomposition of hexadecyl trimethyl ammonium ions $\left(230-290^{\circ} \mathrm{C}\right)$. It is clear that the nanocomposite exhibits higher thermal stability compared with the pure sPS-g-PMMA copolymer.

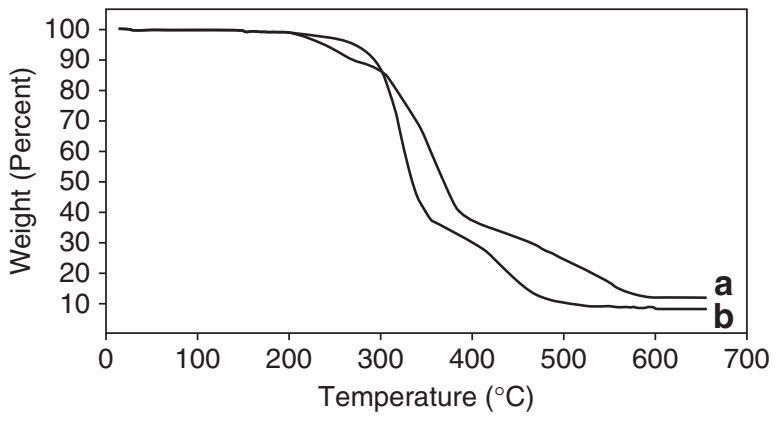

Figure 10 Thermogravimetric analysis of the syndiotactic polystyrene-graftpoly(methyl methacrylate) (sPS-g-PMMA)/montmorillonite nanocomposite (a) and pure sPS-g-PMMA (b).

For instance, the temperature at which $50 \mathrm{wt} \%$ is lost is shifted by ca $36{ }^{\circ} \mathrm{C}$ toward a higher temperature. This enhancement in the thermal properties is due to the homogeneous dispersion of the MMT nanolayers in the sPS-g-PMMA matrix, as depicted in the TEM images, which act as barriers to minimize the permeability of volatile degradation products out of the material.

\section{CONCLUSIONS}

This paper describes an effective route for the graft copolymerization of MMA monomer onto sPS using free-radical polymerization, and the effect of an O-MMT on the final properties of a graft copolymer sample. A VsPSM was synthesized by reacting $\alpha$-ph-ch-sPS with 9decen-1-oxy derived from a 9-decen-1-ol moiety. The resulting multicenter macromonomer was subsequently copolymerized by free-radical polymerization using a heterogeneous process to yield a graft copolymer (sPS- $g$-PMMA) in the presence of MMA monomer. The VsPSM and graft copolymer separated from the reaction mixtures were purified and analyzed. FTIR and ${ }^{1} \mathrm{H}$-NMR studies indicate that these chemical reactions can introduce a vinyl terminal group and PMMA segments into sPS. Additional evidence of the effectiveness of graft copolymerization was also obtained from the DSC data. A study of the thermal properties showed that the presence of graft segments affected the melting behavior and $T_{\mathrm{g}}$ of the sPS backbone. The $T_{\mathrm{g}}$ values of the graft copolymer increased slightly in the presence of PMMA segments by impeding chain flexibility. 
The sPS-g-PMMA/MMT nanocomposite ( $3 \mathrm{wt} \% \mathrm{MMT}$ ) was prepared by a solvent-blending method. The XRD and TEM analyses showed that the sPS-g-PMMA/MMT nanocomposite has an exfoliated structure and that the O-MMT layers were well dispersed in the polymer matrix. Compared with pure sPS-g-PMMA, the nanocomposite showed a much higher decomposition temperature and higher $T_{\mathrm{g}}$. The main reason for the higher thermal stability of the nanocomposite may due to the fact that the MMT layers in the nanocomposite can prevent the diffusion of the decomposed product in the polymer, thus requiring more time and energy for the decomposition process.

\section{ACKNOWLEDGEMENTS}

I express my gratitude to the Bonyade Melli Nokhbeghan Institute and Payame Noor University for supporting this project.

1 Giannakas, A., Spanos, C. G., Kourkoumelis, N., Vaimakis, T. \& Ladavos, A. Preparation, characterization and water barrier properties of PS/organo-montmorillonite nanocomposites. Eur. Polym. J. 44, 3915-3921 (2008).

2 Park, C. I., Choi, W. M., Kim, M. H. \& Park, O. O. Thermal and mechanical properties of syndiotactic polystyrene/organoclay nanocomposites with different microstructures. J. Polym. Sci. Part B Polym. Phys. 42, 1685-1693 (2004).

3 Sorrentino, A. \& Pantani, R. Injection molding of syndiotactic polystyrene/clay nanocomposites. Polm. Eng. Sci. 46, 1768-1777 (2006).

4 Morgan, A. B. Flame retarded polymer layered silicate nanocomposites: a review of commercial and open literature systems. Polym. Adv. Technol. 17, 206-217 (2006).

5 Torre, L., Lelli, G. \& Kenny, J. M. Synthesis and characterization of sPS/montmorillonite nanocomposites. J. Appl. Polym. Sci. 100, 4957-4963 (2006).

6 Tseng, C. R., Lee, H. Y. \& Chang, F. C. Crystallization kinetics and crystallization behavior of syndiotactic polystyrene/clay nanocomposites. J. Polym. Sci. Part B Polym. Phys. 39, 2097-2107 (2001).

7 Sain, S. \& Khatua, B. B. Synthesis of highly exfoliated PS/Na+ ${ }^{-}$MMT nanocomposites by suspension polymerization using $\mathrm{Na}^{+}-\mathrm{MMT}$ clay platelets as suspension stabilizer. Macromol. Res. 19, 44-52 (2011).

8 Yoon, K. B., Sung, H. D., Hwang, Y. Y., Noh, S. K. \& Lee, D. H. Modification of montmorillonite with oligomeric amine derivatives for polymer nanocomposite preparation. Appl. Clay. Sci. 38, 1-8 (2007).

9 Chigwada, G., Wang, D., Jiang, D. D. \& Wilkie, C. A. Styrenic nanocomposites prepared using a novel biphenyl-containing modified clay. Polym. Degrad. Stabil. 91, 755-762 (2006).

10 Jaymand, M. Surface modification of montmorillonite with novel modifier and preparation of polystyrene/montmorillonite nanocomposite by in situ radical polymerization. J. Polym. Res. 18, 957-963 (2011).

11 Yuan, W., Guo, M., Miao, Z. \& Liu, Y. Influence of maleic anhydride grafted polypropylene on the dispersion of clay in polypropylene/clay nanocomposites. Polym. J. 42, 745-751 (2010)

12 Vaia, R. A. \& Giannelis, E. P. Lattice of polymer melt intercalation in organicallymodified layered silicates. Macromolecules 30, 7990-7999 (1997).

13 Jaymand, M. Synthesis and characterization of an exfoliated modified syndiotactic polystyrene/Mg-Al-layered double-hydroxide nanocomposite. Polym. J. 43, 186-193 (2011).

14 Morgana, A. B. \& Harrisb, J. D. Exfoliated polystyrene-clay nanocomposites synthesized by solvent blending with sonication. Polymer 45, 8695-8703 (2004).

15 Tseng, C. R., Wu, S. C., Wu, J. J. \& Chang, F. C. Crystallization behavior of syndiotactic polystyrene nanocomposites for melt- and cold-crystallizations. J. Appl. Polym. Sci. 86, 2492-2501 (2002).

16 Wang, Z. M., Chung, T. C., Gilman, J. W. \& Manias, E. Melt-processable syndiotactic polystyrene/montmorillonite nanocomposites. J. Polym. Sci. Part B Polym. Phys. 41, 3173-3187 (2003).

17 Shen, Z., Zhu, F., Liu, D., Zeng, X. \& Lin, S. Preparation of syndiotactic polystyrene/ montmorillonite nanocomposites via in situ intercalative polymerization of styrene with monotitanocene catalyst. J. Appl. Polym. Sci. 95, 1412-1417 (2005).

18 Bruzaud, S., Grohens, Y., Ilinca, S. \& Carpentier, J. F. Syndiotactic polystyrene/ organoclay nanocomposites: synthesis via in situ coordination-insertion polymeri- zation and preliminary characterization. Macromol. Mater. Eng. 290, 1106-1114 (2005).

19 Sengupta, R., Chakraborty, S., Bandyopadhyay, S., Dasgupta, S., Mukhopadhyay, R., Auddy, K. \& Deuri, A. S. A short review on rubber/clay nanocomposites with emphasis on mechanical properties. Polym. Eng. Sci. 47, 1956-1974 (2007).

$20 \mathrm{Li}$, J., Zhou, C. \& Gang, W. Study on nonisothermal crystallization of maleic anhydride grafted polypropylene/montmorillonite nanocomposite. Polym. Test. 22, 217-223 (2003).

21 Alexandre, M., Dubois, P., Sun, T., Garces, I. M. \& Jerome, R. Polyethylene-layered silicate nanocomposites prepared by the polymerization filling technique: synthesis and mechanical properties. Polymer 43, 2123-2132 (2002).

22 Kim, M. H., Park, C. I., Choi, W. M., Lee, J. W., Lim, J. G., Park, O. O. \& Kim, J. M. Synthesis and material properties of syndiotactic polystyrene/organophilic clay nanocomposites. J. Appl. Polym. Sci. 92, 2144-2150 (2004).

23 Samakande, A., Hartmann, P. C., Cloete, V. \& Sanderson, R. D. Use of acrylic based surfmers for the preparation of exfoliated polystyrene/clay nanocomposites. Polymer 48, 1490-1499 (2007).

24 Ishihara, N., Seimiya, T., Kuramoto, M. \& Uoi, M. Crystalline syndiotactic polystyrene. Macromolecules 19, 2464-2465 (1986).

25 Gowd, E. B., Tashiro, K. \& Ramesh, C. Structural phase transitions of syndiotactic polystyrene. Prog. Polym. Sci. 34, 280-315 (2009).

26 Schellenberg, J. Recent transition metal catalysts for syndiotactic polystyrene. Prog. Polym. Sci. 34, 688-718 (2009).

27 Grassi, A., Caprio, M., Zambelli, A. \& Bowen, D. E. Synthesis and characterization of syndiotactic styrene-ethylene block copolymers. Macromolecules 33, 8130-8135 (2000).

28 Pellecchia, C., Pappalardo, D., D'Arco, M. \& Zambelli, A. Alternating, ethylene-styrene copolymerization with a methylaluminoxane-free half-titanocene catalyst. Macromolecules 29, 1158-1162 (1996)

$29 \mathrm{Li}$, H. M., Shen, Z. G., Zhu, F. M. \& Lin, S. A. Polymer blends of sPS/PA6 compatibilized by sulfonated syndiotactic polystyrene. Eur. Polym. J. 38, 1255-1263 (2002).

$30 \mathrm{Gao}$, Y. \& Li, H. M. Synthesis and characterization of acetylated syndiotactic polystyrene. Polym. Int. 53, 1436-1441 (2004).

$31 \mathrm{Li}$, H. M., Chen, H. B., Shen, Z. G. \& Lin, S. A. Preparation and characterization of maleic anhydride-functionalized syndiotactic polystyrene. Polymer 43, 5455-5461 (2002).

32 Stack, S., O'Donoghue, O. \& Birkinshaw, C. The thermal stability and thermal degradation of blends of syndiotactic polystyrene and polyphenylene ether. Polym. Degrad. Stabl. 79, 29-36 (2003).

33 Gao, Y. \& Li, H. M. Synthesis of syndiotactic-polystyrene-graft-poly(methyl methacrylate) and syndiotactic-polystyrene-graft-atactic-polystyrene by atom transfer radical polymerization. Eur. Polym. J. 41, 2329-2334 (2005).

34 Dong, J. Y., Manias, E. \& Chung, T. C. Functionalized syndiotactic polystyrene polymers prepared by the combination of metallocene catalyst and borane comonomer. Macromolecules 35, 3439-3447 (2002).

35 Tomita, K. \& Ono, T. Synthesis of polyaspartate macromonomer having a vinyl end group and application to dispersion copolymerization of styrene. Colloid. Polym. Sci. 287, 109-113 (2009).

36 Ohnaga, T. \& Sato, T. Influences of substrate surfaces on the band formation behavior in an oriented thermotropic liquid crystalline polyester. Polymer 37, 3799-3803 (1996).

37 Bonilla-Cruz, J., Sánchez, C., Schubert, U. \& Saldívar, G. Controlled 'grafting-from' of poly [styrene-co-maleic anhydride] onto polydienes using nitroxide chemistry. Eur. Polym. J. 46, 298-312 (2010).

38 Villegas, A. G., Ocampo, M. A., Luna-Bárcenas, G. \& Saldívar-Guerra, E. Obtainment of graded index preforms by combined frontal co-polymerization of MMA and BzMA. Macromol. Symp. 283, 336-341 (2009).

39 Jaymand, M. Synthesis and characterization of conductive polyaniline-modified polymers via nitroxide mediated radical polymerization. Polymer (Korea) 34, 553-559 (2010).

40 Kim, S. Y., Lee, K., Jung, H., Shim, S. E., Lee, B. H. \& Choe, S. Macromonomers having different molecular weights of polyethylene glycol and end group functionalities in dispersion polymerization of styrene. Polymer 46, 7974-7981 (2005).

41 Yilmaz, F., Cianga, I. Ito, K., Senyo, T. \& Yagci, Y. Synthesis and characterization of $\alpha, \omega$-heterofunctional poly(ethylene oxide) macromonomers. Macromol. Rapid Commun. 24, 316-319 (2003).

42 Reichert, P., Nitz, H., Klinke, S., Brandsch, R., Thomann, R. \& Miilhaupt, R. Morphological stability of poly(propylene) nanocomposites. Macromol. Mater. Eng. 5, 17-27 (2000).

43 Wang, K., Liang, S., Du, R., Zhang, Q. \& Fu, Q. The interplay of thermodynamics and shear on the dispersion of polymer nanocomposite. Polymer 45, 7953-7960 (2004). 